\title{
A physical notice board with digital logic and display
}

\author{
Nicolas Villar, Kristof Van Laerhoven, Hans Gellersen \\ Embedded Interactive Systems Group \\ Computing Department \\ Lancaster University, UK \\ +44 (0) 1524594801 \\ \{villar, kristof, hwg\}@comp.lancs.ac.uk
}

\begin{abstract}
A physical notice board is augmented with digital capabilities to provide additional functionality. The notice board retains its form factor, and when used with interactive pins with controllable lights it can be used to signal to the user information about the state of documents posted on the board. Both board and augmented pins are easy to deploy and cheap to produce.
\end{abstract}

\section{Keywords}

Notice boards, interactive surfaces, physical interfaces, Pin\&Play

\section{INTRODUCTION}

We have designed a digitally augmented notice board based on the Pin\&Play system. It demonstrates our idea for a novel interface based on the metaphor of pinning and removing papers from the surface of a notice board. It also reflects an interesting extension of the functionality of the physical object when it is overlaid with digital capabilities.

The notice board allows notes and documents to be attached onto it by using pins. The pins are colored either red, orange or green - corresponding to three different levels of priority one would assign to a document that was posted on a notice board. This allows a way for the user of the notice board to inform the system when she would like to be reminded of the existence of the document, and the need to pay attention to what it contains. As such, it allows the user to attach a digital reminder to a physical document, in the same way that it is possible to set a reminder to do a certain task on a PDA or a calendar on the PC.

In this way the user can choose how much time they would like to pass before they are reminded of the document by choosing a pin designated with a high, medium or low priority color. When this time expires, a small light built onto the pin will begin to flash to display this fact. The user must then unfasten the document from the board in order for the pin to stop flashing. Information about when documents were posted, their level of priority and how much time they have before their reminder expires is displayed on a screen nearby. This information can also be accessed via a web interface, and the system can deliver the reminders via messages to the user's e-mail inbox.

\section{MOTIVATION}

Notice Boards as Information Displays

Notice boards are generally made of cork or other soft materials that allow pins to be fastened onto them, allowing papers and other objects that allow it to be attached onto a vertical surface. These boards are often placed in very visible places where people spend time or walk by often. They vary is size and are used in a variety of locations, and the simple functionality they provide is put to a wide range of uses - from displaying family photographs to posting information to others to see.

A particular application of notice boards is to use them to post up documents that need be dealt with in some way. Pinning a document on the board creates a reminder that attention needs to be paid to the particular piece information of paper by making it highly visible. For example, an electricity bill that needs to be paid in the following week, or a personal letter that should be answered.

We became interested in considering how people use notice boards as a way to organize their way in how to deal with paper-based documents. This motivated us to think about how we could extend and increment the functionality of traditional notice boards to enable more powerful applications.

\section{Pin\&Play}

Pin\&Play is a system that allows individual objects to be powered and networked together by placing them on a common surface [1]. It allows physical nodes to be added and removed in an ad-hoc fashion. These nodes consist of a variety of interaction components, such as lights, switches, sliders and dials. Each node also contains some 
memory, and the ability to retain this memory for several years, even when disconnected from the networked surface.

The surface provides an interface to a controller on which applications can be developed to create a logical overlay between the nodes. This is done via conductive fabrics embedded within insulating layers of the surface. The controller can be a local or remote networked PC, or a small embedded device. When running on a PC, the controller can also act as graphical user interface to the networked nodes - reacting to their state or controlling their behavior. It also provides remote access and control of the surface through a web interface.

In the current implementation of Pin\&Play, nodes are attached onto the surface by the use of pins that allow them to pierce and fasten onto it. Different sections of the pins make a physical connection with conductive layers embedded within the surface, which in turn provides a physical network layer on which runs the industrystandard 1-Wire protocol [2]. The pins provide a bus on the node onto which small devices can be connected, networked and powered, allowing the creation of very small interaction components (Figure 2).

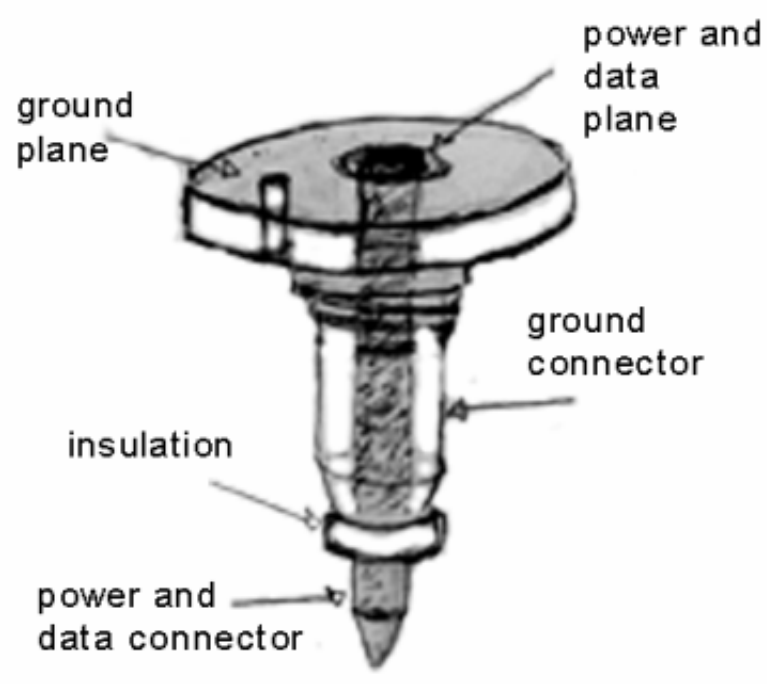

Figure 1. A Pin\&Play node

The pin connectors add an interesting affordance to the interface, as the majority of people are familiar with the concept of pinning objects onto surfaces. We believe that this will allow for the development of intuitive physical interfaces that can be digitally augmented to allow for complex interactions.

\section{DESCRIPTION OF DEMONSTRATION}

The demonstration consists of an augmented notice board that retains the appearance and functionality of a traditional notice board that one might find hanging on a kitchen wall or office. The only clue as to its digital augmentation is the presence of a thin cable emerging from one corner, which connects it to a laptop nearby.

There are also a set of objects in the form factor of tacks, or pushpins. They are made to closely resemble the types of pins one would use to post documents on a notice board. They have a flat head with a $1.5 \mathrm{~cm}$ diameter and a pin protruding from the middle. On the opposite side, the pin contains a small blue LED lamp (Figure 2).

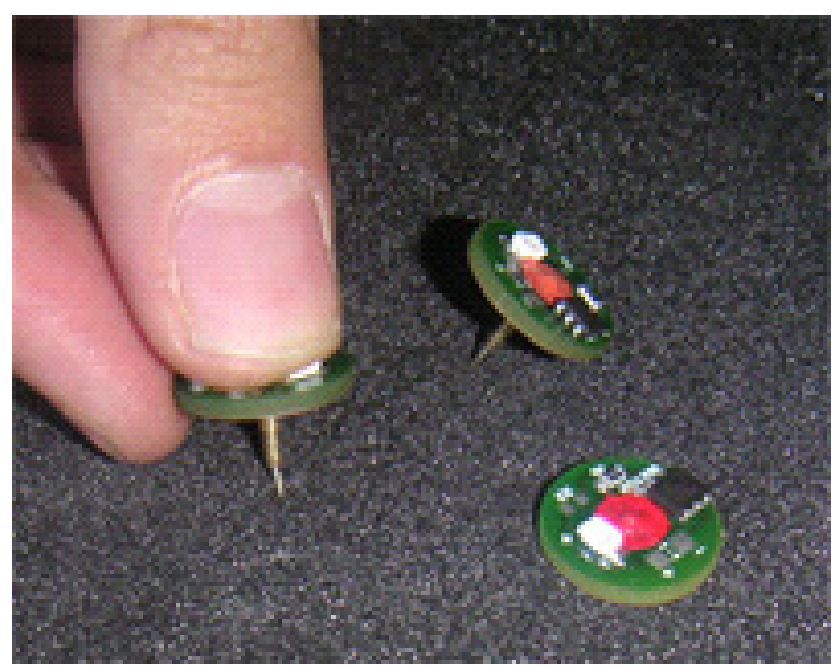

Figure 2. The Pin\&Play notice board pins

The pins are divided into three differently colored groups: red, orange and green. Pins from each group represent a reminder to deal with a document in a high, medium or low priority manner. If a red/high priority pin is used to fasten a document, a reminder is set for a day's time. An orange/medium priority pin has a time of one week, and a green/low priority a time of one month. The associated time to any of the categories of pins can be configured, and it is also possible to introduce other categories with new associated meanings.

A graphical interface presented on the screen of the laptop shows an abstracted representation of the current documents posted on the board (Figure 3). They are stamped with the time and date when they were originally posted, when the reminder is set to trigger the alarm, and a countdown of how much time is remaining on the reminder. This interface can also be accessed remotely by visiting a website. 


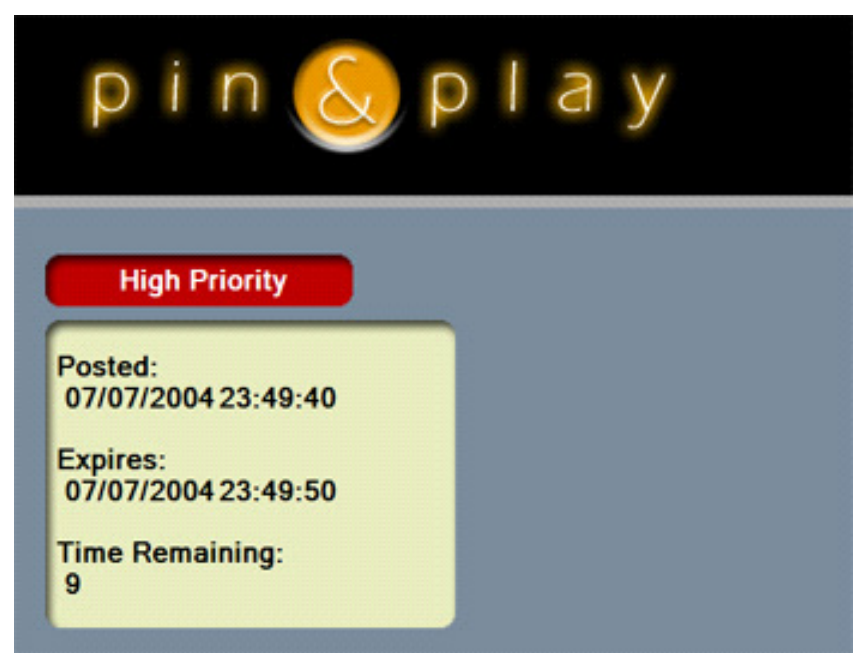

Figure 3. Digital representation

When the time for the digital reminder is reached, a document is flagged as expired. This fact is displayed on the physical notice board by turning on a small but bright blue LED on the pin of the appropriate document.
The light on the pin is highly visible, and clearly stands out of other objects on the notice board. In this way, a suggestion is made to the user to pay attention to the attached document in a non-obtrusive and highly relevant way. To reset the alarm, the pin must be unfastened. The document may be attached again with the same or a different category pin, similar to pressing Snooze on an alarm clock.. Hopefully though, the document will be dealt with or at least taken off the notice to make room for new posts.

\section{REFERENCES}

1. K. Van Laerhoven, N. Villar, A. Schmidt, H.-W. Gellersen, M. Håkansson, and L. E. Holmquist. "Pin\&Play: The Surface as Network Medium" In IEEE Communications Magazine, April 2003, Vol.41 No.4., ISSN: 0163-6804, IEEE Press, pp. 90-96.

2. "Technical Brief: 1-Wire Net Design Guide," Dallas Semiconductors, 2002. 